\title{
Lumbar Disc Injury: Mechanisms and Intervention Options
}

\author{
Jim Schilling ${ }^{\mathrm{a}}$, Jupil Ko ${ }^{\mathrm{b}}$, \& Seung-Taek Lim, \\ ${ }^{a}$ Associate Clinical Professor, Northern Arizona University, Phoenix AZ, U.S \\ ${ }^{b}$ Assistant Professor, Division of Health \& Kinesiology, Incheon National University, Incheon, Republic of Korea \\ ${ }^{c}$ Research Professor, Olympic Studies Center, Kangwon National University, Gangwon-do, Republic of Korea
}

\begin{abstract}
Low back pain is prevalent in sport and the lumbar disc is a pain generator. Conservative care of these physically demanding patients is not consistent as outcome data has not established optimal strategies. Investigating disc injury mechanisms using experimental models will contribute to the creation of treatment strategies that will take into effect movements to avoid and minimize to reduce unwanted strain on the injured tissues throughout the recovery process. Additionally, how disc tissue acquires nutrition would help provide insight into activities that may facilitate the ability of disc tissue to sustain health and promote optimal healing. Rotational forces and compression in trunk-flexed positions may inflict injury to the annulus and endplate structures. Activity to facilitate nutrients through the endplate into the nucleus of the disc and restriction of exasperating movements may be effective in returning athletes to their desired levels of performance efficiently. The purpose of this review is to inquire possible disc injury mechanisms and how they occur in sport. It will also address recommendations for treatment interventions based on disc healing and metabolism evidence.
\end{abstract}

Key words: lumbar disk, injury mechanisms, pain, movements, sport

\section{Introduction}

Low back pain is ranked first as a cause of disability (Ehrlich, 2003) and affects 84\% of adults once in their life (Beeckmans et al., 2016). Return to sport guidelines for disc injured athletes is lacking and would be sport and position specific. A study done by the National Football League over a 12-year span found a rise in disc herniation prevalence and on average, players missed over half a season of competition. (Gray BL

Submitted : 18 May 2021

Revised : 1 July 2021

Accepted : 5 October 2021

Correspondence : limdotor@gmail.com et al., 2013) Disc injuries occur in sport and when they do athletes are subject to significant time loss and possible reoccurrence. The outer annular layers of a lumbar disc are innervated and a potential pain generator (Bogduk et al., 1981), along with the endplates (Wang et al., 2012). Most lumbar disc injuries are located in the posterolateral portion of the annulus (Lurie et al., 2014) and primarily occur at L4-5, L5-S1 segments (Wang et al., 2013). Disc injuries are consistent with sports that require athletic movements of rotation and or side bending in flexed positions (Dowdell et al., 2018). Disc tears or fissures have been subcategorized into 3 types (Fardon et al., 2014). The first is called 
a transverse fissure which occurs when outer annular layers tear off the vertebral body. A second is called a concentric fissure which results from the annular layers separating from each other and nuclear material is allowed to filtrate between them. The third is called a radial fissure, usually vertically, from the inner to outer annular layers of the disc. Athletic movements and resultant external forces placed on intervertebral discs of the lumbar spine leave them at risk of injury.

Understanding the mechanisms that create injury experimentally may provide valuable insight into treatment strategies for returning athletes to competition safely and less likely for reinjury. In addition, becoming familiar with how the disc is provided nutrition to maintain tissue health will contribute to strategies to facilitate disc healing efficiency. The purpose of this paper is to review the experimental evidence specific to disc injury mechanisms using models and provide examples of how these positions and loads occur in the lumbar spine during sport. In addition, we will suggest a progression of relevant treatment interventions that are effective and efficient for the recovery of athletic disc injuries.

\section{Literature review}

\section{Disc Injury Mechanism}

Single plane movements of spinal segments have been studied for disc injury responses. Trunk rotational movements are shown to cause transverse fissures, possibly secondary to shear forces created when torsion continues at bony end-range but rotation has not been shown to cause radial fissures of the disc in isolation with experimentation (Adams \& Dolan, 2016). Posterior and posterior-lateral disc herniations occurred in porcine spines with high numbers of cyclic flexion and extension with low-magnitude axial compression loads and more frequent with high loads (Callaghan \& McGill, 2001). Cyclic movements with compression also caused inner annular fiber injury, which may be linked to disc annular fatigue and loss of stiffness (Noguchi et al., 2016). Multiplanar movements have also been investigated for disc injury using a computer model (Schmidt et al., 2007). The results suggest the annulus is under highest strain in the posterolateral region, further supporting the most common location of lumbar disc herniations (Schmidt et al., 2007). A disc may herniate under a combination of rotation and lateral bending, rotation and flexion, or lateral bending and flexion movements. The risk of injury is significantly increased when an axial load is also added (Schmidt et al., 2007).

Providing a load on the annular layers of ovine discs were inflicted by increasing intradiscal pressure. A gradual increase in pressure within a neutral positioned segment by injecting a gel into the nucleus caused radial fissures posteriorly through a separation of annular layers (Concentric fissure) and failure to the outer posterior region possibly due to inter-annular cohesion weakness (Veres et al., 2008). A similar study using a gradual increase in pressure in discs of segments flexed $7^{\circ}$ created an increase in central-posterior radial fissures and fractures at the mid-annulus or endplate ridge apex along with an increase in herniations compared to a neutral position (Veres et al., 2009). The magnitude of these experimental pressures have been attained with the compression force of football blocking (Gatt et al., 1997). Another study using a gradual increase in pressure and application of rotational forces in flexion was found to reduce the occurrence of radial fissures of the annulus in isolation but facilitated radial fissures that involved an endplate fracture adjacent to the endplate ridge apex (Veres et al., 2010). The annular fibers in the direction of rotation would experience higher axial tensile load (every other layer) creating an uneven load-sharing situation when the nucleus is pressurized increasing stress at the endplate junction and failure at mid-annulus, referred to as the endplate ridge apex (Veres et al., 2010a). Sudden, high-pressure impulse internal loading, similar to a contact or collision load, was applied in a neutral position and caused 
central-posterior radial fissures and injury of the cartilaginous endplate at the inner-annulus (Veres et al., 2010b). In theory, there was not enough time for inter-annular fluid flow to occur through pores in the annular layers to decrease hydrostatic pressure within the nucleus (Veres et al., 2010b). When segments were positioned in flexion, there was an increase in endplate fractures (Veres et al., 2010b).

Experimental conditions providing axial compression loads on ovine discs were also created. When analyzing injury to ovine discs at varying compression rates (2 and $40 \mathrm{~mm} / \mathrm{min}$ ) in neutral and flexion, annular failure and herniation required positioning in flexion and a high compression rate (Wade et al., 2014). Flexion without compression did not provide enough stress to the annulus to cause injury. The annular fissure was initiated adjacent to the endplate ridge apex creating a situation where the inner annular layers are unable to withstand the nuclear pressure and tear allowing the nucleus to lacerate the outer layers and eventually herniate (Wade et al., 2014). The oblique angle of the annular fibers increases from the outer to inner portions making mid to outer layers most stressed with flexion (Wade et al., 2014). With only inner annular layers injured, it is possible that the early phases of disc injury could be asymptomatic if the endplate is not involved. Another study using flexion and high compression rates $(40 \mathrm{~mm} / \mathrm{min})$ found anterior shear and displacement of the superior vertebrae resulting in a load on the oblique annular fibers in the same direction and at the same time relaxing counter-oblique oriented fibers allowing for disruption of lateral fibers with an increase in hydrostatic pressure, forcing nuclear material laterally (van Heeswijk et al., 2017). Once migrated into the outer layers, the weak intra-annular bond between layers allows for a concentric fissure to migrate to the posterior-lateral region of commonly found herniations. When non-degenerated human cadaver discs were placed in either normal lordosis or flexed positions to where the endplates were parallel and compressed to failure, vertebral bodies fractured first with normal lordosis and endplates first in a flexed position with less than half of the axial load (Curry et al., 2016).

A sudden, ultra-high rate of compression loading $(400 \mathrm{~mm} / \mathrm{min})$, as encountered in a contact or collision sport, in flexion found $83 \%$ of the segments suffered herniations and $70 \%$ a rupture at the annulus-endplate junction (Wade et al., 2015) which is comparable to the $65 \%$ endplate junction failure found clinically with disc patients (Rajasekaran et al., 2013). The annular layers will experience the highest strain at the endplate ridge apex and tear, further compression leads to herniation (Wade et al., 2015). With the segment in a flexed position, the oblique annular fibers in the direction of flexion are stretched and the counter-oblique fibers are relaxed creating weak points in the layers presenting a pathway for posterior-lateral nuclear migration and herniation (Wade et al., 2015).

When compression is applied to discs in complex positions, such as a postural combination of $0-13^{\circ}$ flexion, $0-10^{\circ}$ lateral flexion, $0-4^{\circ}$ rotation and $0-800 \mathrm{~N}$ compression with a $2 \mathrm{~Hz}$ cycle, $76 \%$ endplate junction fractures and $24 \%$ outer-posterior annulus injury occurred (Berger-Roscher et al., 2017). It appears flexion and lateral flexion are factors for endplate fractures and rotation increases the risk. It was suggested the "crackling" sound often reported from the low back by patients may be caused by an endplate junction fracture (Berger-Roscher et al., 2017). Prepositioned segments in $7^{\circ}$ of flexion, $20^{\circ}$ of laterally flexion, and $5^{\circ}$ of rotation and submitted to high compression rates $(40 \mathrm{~mm} / \mathrm{min}$.) demonstrated most discs suffered from mid-annular alternate layer damage and nuclear material extruded into the area via annular rupture and circumferential tracking posterior-laterally (Wade et al., 2017). A disc in a complex posture significantly reduces its ability to withstand compressive loading without failure (Wade et al., 2017).

Sudden compression in complex postures such as a segment prepositioned in $7^{\circ}$ of flexion, $20^{\circ}$ of laterally flexion, and $5^{\circ}$ of rotation and subjected to ultra-high compression rates $(400 \mathrm{~mm} / \mathrm{min}$.) on the disc resulted 
in an anterior shear of the superior vertebrae and inability for nuclear material to infiltrate through the annulus (Shan et al., 2017). Ultra-high compression rates produced more direct annular fissures with circumferential tracking to the posterior-lateral region along with endplate fractures than lower compression rates (Shan et al., 2017). With sudden compression there is less time for nuclear fluid to equalize within the disc. The high incidence of endplate fractures is caused by increased hydrostatic pressure in the nucleus and an increase in tensile load on the annular fibers with disc sudden forceful distraction. An increase in endplate-annulus junction tears suggests complex postures play a significant role in herniations.

\section{Movement and Annular Pressure}

Transverse trunk movements that involve high torsion loads may create transverse fissures but rotation alone does not cause radial fissures with experimentation (Adams et al., 2010). Forceful lumbar torsion could cause a shear load on the annular layers due to $90^{\circ}$ facet orientation causing annular fiber avulsion and possibly endplate fracture. Cyclical sagittal movement may also be a mechanism for inner annular injury (Noguchi et al., 2016) and possible disc herniation (Callaghan et al., 2001). Multiplanar movements may be a risk factor for disc herniation, especially with the addition of an axial load (Schmidt et al., 2007). All these movements are feasible for disc injury and required in most sport events.

When nuclear pressure was increased in discs to discover the annular response, concentric fissures occurred in neutral positions (Veres et al., 2008) with radial fissures and endplate fractures being observed in flexed positions (Veres et al., 2009). Rotation was also found to create endplate fractures, possibly due to unequal load from alternating directions of annular fibers (Veres et al., 2010a). Abrupt increases in internal pressure in neutral positions creates radial fissures with endplate fractures, possibly due to the inability of the nuclear matter to infiltrate through the annular layers creating pressures that the disc tissues are unable to withstand (Veres et al., 2010b). Sudden compression loads in collision sports may meet the forces necessary for injuries sustained in these experimental trials.

An experiment was administered using external forces to create a compression load on discs, annular failure at the endplate ridge apex and herniation occurred with high compression rates (Wade et al., 2014). The experiment suggests radial fissures may initiate at the endplate ridge apex, where the annulus is narrowest and advance inward. With the inner annulus lacking nociceptive input, acute radial fissure injury will likely be asymptomatic. A similar study witnessed an anterior translation of the superior vertebrae and unequal tensile load of annular fibers resulting in lateral radial fissures (van Heeswijk et al., 2017). If normal lordosis is removed and compression forces are applied with parallel endplates, endplate fractures result (Curry et al., 2016). This mechanism could come into play with football if compression loads are sustained in trunk flexion, although unlikely with lordosis being well-maintained.

Sudden axial compression, similar to the mechanism encountered with a collision in sport, an endplate fracture may occur due to an increase in nuclear hydrostatic pressure causing an abrupt, significant increase in tensile load on the annular fibers in addition to disc herniation (Wade et al., 2015; Shan et al., 2017). An athlete who sustains a lumbar injury by a sudden, high axial compression load is likely to have an endplate fracture at minimum.

When segments are placed in complex postures with compression, it appears flexion and lateral flexion are needed for endplate fractures to occur. If rotation is added, it significantly increases the chances.23 If a patient reports a "crackling” or "snapping” sound or feel with an acute lumbar injury, it could be an indication of an endplate fracturing (Berger-Roscher et al., 2017) 


\section{Mechanisms By Tissue}

Certain mechanical loads placed on experimental discs result in a variety of injury consequences. With anatomical limitations of rotation in the lumbar region ( $90^{\circ}$ facet orientation), forceful torsion could create shear forces on the annular fibers causing avulsion at the endplate but not necessarily radial fissures (Adams \& Dolan, 2016). Repeated sagittal plane movement with compression loads may cause inner annular injury secondary to annular fiber fatigue (Noguchi et al., 2016). If high rates of compression are encountered with the segments fixated in flexed positions, there seems to be an injury progression that occurs where initially the mid-annulus injures at the endplate ridge apex, next the inner annulus fails, then there is endplate avulsion, and finally herniation (Wade et al., 2014). If anterior shear of the superior vertebrae occurs with compression while flexed, nuclear material migrates laterally until it reaches the outer annular layers and circumferentially tracks to the posterior-lateral region (van Heeswijk et al., 2017).

There are several mechanisms identified that place risk of endplate fracture that are of value to recognize. Torsional forces can create high tensile loads on alternating annular layers producing stress at the annular-endplate junction, resulting in endplate fractures (Veres et al., 2010a). If cyclical axial compression loads are placed on the spine in complex postures, endplate junction fractures resulted (Berger-Roscher et al., 2017) and, although unlikely in sport, if lordosis was removed with compression they also occurred (Curry et al., 2016). Sudden, high compression loads may also resulted in an endplate fracture, possibly due to an immediate increase in nuclear hydrostatic pressure from lack of time to dissipate pressure (Wade et al., 2015) and a "crackling" may be sensed or heard (Berger-Roscher et al., 2017).

\section{Application of Mechanisms in Sport}

In this section we will demonstrate how these experimental mechanisms apply to movements and loads created by demands in a few sports. A significantly higher percentage of college baseball players have encountered DDD than nonathletes. (Hangai et al., 2009) In baseball, pitching and batting movements result in repeated large axial rotations using high angular accelerations (Fleisig et al., 2013) which may place an accumulating load on disc annular fibers. With pitching, excessive arm extension occurs with early cocking which increases trunk side bending followed by trunk flexion and rotation. Although angular velocities at the trunk may not be exceedingly high, the multiplanar movement has been suggested as a mechanism for disc injury. Batting does involve high rotational accelerations and repeated high end-range loading placing annular fibers at risk of shear forces. Movement in a golf swing provides the suggested planes where disc lesions have been found to be vulnerable, flexion, side bending and rotation, all in one swing. (Lindsey \& Vandervoort, 2014) A 5-year epidemiology study with tennis found the lumbar spine region to have the greatest number of injuries. (Fu et al., 2018) The lumbar disc was determined to be a common tissue for injury in tennis resulting from high axial rotation loads. (Dines et al., 2015) The tennis serve requires side bending with flexion and groundstrokes require trunk rotation and end-range loading varying on stance positions. An epidemiology study in volleyball reported a high prevalence of DDD. (Kulling et al., 2014) During a spiking maneuver or jump serve, movement required in the trunk is flexion, side bending and rotation placing a disc vulnerable. Bowling is also a sport which produces a high number of lumbar disc herniations possibly from flexion, lateral flexion, or rotation and compression loads. (Mundt et al., 1993) In football, an epidemiology study found $76 \%$ of all disc herniations were in the lumbar spine. (Gray et al., 2013) Extremely high axial compressive loads from blocking along with shear forces from blocking and tackling and lateral flexion may be the primary mechanisms.

Most sports require repeated axial rotation of the 
trunk with forceful loads at end-range and often in a flexed position. Overhead movements such as pitching in baseball, spiking in volleyball, or serving in tennis require the trunk to go from an extended position to flexion with side bending. A golf swing is an athletic movement placing the disc in all predisposing planes of annular injury suggested by experimentation. Players in football can be exposed to compression forces in tackling and blocking which create high hydrostatic pressure resulting in disc herniations and endplate fractures. Sport participation places lumbar discs at risk whether it be the movement, compression loads, repeated end-range loads, or a combination making lumbar disc injury prevention and conservative treatment strategy effectiveness critical.

\section{Disc Injury Intervention}

Athletes with disc injuries from 1990-2009 either discontinued sporting activity or received a discectomy (Iwamoto et al., 2010). Both approaches seemed satisfactory in returning athletes to their original levels of sports activities (Iwamoto et al., 2010). Professional athletes with disc herniation had no significant difference in outcomes between surgery or no surgery (Hsu et al., 2011). These outcome studies suggest conservative care is as effective as surgical procedures even without providing specific treatment strategies.

A recommended progression (4 Phase) for the treatment of lumbar disc injuries in athletes is provided a based-on literature inquiring disc healing, nutrient transfer and load adaptation. Disc healing is restricted due to a low cell density and extensive mechanical loading (Adams \& Dolan, 2016). With a limited blood supply, the cells of the disc can survive at very low levels of oxygen but need significant levels of glucose (Hughes et al., 2012). Nutrition to the cells of the disc is dependent on the transfer of nutrients from blood vessels located at the outer annulus and through the endplates by diffusion (Hughes et al., 2012).

The cells of the outer annular layers are similar to tendon and ligament and appear to have a similar healing process (Ahlgren et al., 2000). Consequently, during the acute stage of healing limited motion may be effective, as with the annular healing of sheep disc (Latham et al., 1994). Pressure is placed on the annular layers from the nucleus at its greatest in the morning, which should be addressed though dehydration strategies (Adams et al., 1987). During the initial phase of lumbar disc injury recovery for an athlete, emphasis should be placed on restricted movement, maintaining lumbar lordosis and disc dehydration. Early phase interventions include dehydrating the disc in the morning with shallow lumbar extensions. Ergonomic positions such as a pillow in the low back to emphasize extension and raising the seat to lesson hip flexion and lumbar flexion. Taping the lumbar region using a technique to restrict lumbar flexion as a feedback mechanism to provide lumbar flexion restraint can also be employed.

During the second phase, a repeated tensile load may increase fibroblast proliferation and stimulate collagen synthesis (Lee et al., 2004). Cyclic, gentle flexion of the spine performed in side-lying to minimize compressive loading could stimulate repair of the outer posterior annulus (Tampier et al., 2007). Rotation of the spine applies tension to alternating annular layers (Krismer et al., 1996), consequently, intersegmental mobilization may facilitate inter-laminae movement and minimize adhesion formation and discogenic pain while facilitating nutrient transfer. A single session of joint mobilization significantly increased fluid diffusion with degenerated nucleus pulposus discs (Beattie et al., 2009). With a radial fissure, begin mobilization techniques in the direction that decreases pain to facilitate inter-annular movement (Adams et al., 2010). After a few days, scar formation develops annular mobilization techniques could begin in the direction causing pain to promote remodeling of the fibrous tissue (Adams et al., 2010). Alternating full lumbar extension and flexion static positions may facilitate fluid diffusion by fluctuating hydrostatic pressures (Adams \& Hutton, 
1983). Either mechanical or manual intermittent traction could also be performed at this stage to provide cyclic loading to facilitate disc metabolism. After pain free movement is experienced, the third phase may begin with motor control training for segmental stability can begin. This is meant to target deep abdominal muscle activation to assist in uni-segmental stability of the lumbar region and shear force restraint. After motor control training is established, McKenzie exercises for repeated lumbar extension and disc nutrition followed by trunk stability training to improve external load restraint may begin. Additional considerations are facilitating rotational movement in the thoracic region and internal rotation of the lead hip to minimize rotational torque forces in the lumbar region.

Discs rely on diffusion to transfer nutrients, so fluctuating changes in intradiscal pressure are important to disc health (Kingsley et al., 2012). A study assessing disc pressures found overnight, disc volume increased $10.6 \%$ in volume and walking 8 hours did not decrease disc volume 10.6\% (Malko et al., 2002). Running at $70 \%$ heart rate maximum for 30 minutes, decreased lumbar disc volume $6.9 \%$, due to an increase in hydrostatic pressure in the disc (Kingsley et al., 2012). Running increased extracellular matrix production and cell proliferation in lumbar discs of rats (Brisby et al., 2010). Walking and low intensity running provide moderate mechanical loading that strengthens tissues by adaptive remodelling (Videman et al., 2010). A systematic review suggested running may have a protective effect on disc (Trompeter et al., 2017). When comparing the effects of dynamic and static compression on the transport and metabolism of oxygen and lactate in and out of the disc, dynamic compression increased oxygen concentration along with decreasing lactate while static compression produced an inverse effect (Huang \& Gu, 2008).

Consistent with these findings of metabolic transfer in discs, the last phase suggests interventions that provide a graded increase in repetition and compression loading may facilitate disc metabolism safely and effectively and the combination compression-decompression mechanism in the disc allows for annular adaptation to hydrostatic pressures within the disc. Examples would include beginning with walking progressing to jogging and finally running, all with graded durations.

When to allow a disc injured athlete to return to sport is a difficult decision as evidence to support specific measures to accurately determine a safe return without reoccurring symptoms are lacking. Six weeks of healing was enough time for sheep discs to withstand a high hydrostatic pressure (Ahlgren, 2000) may be a timeline to consider.

Discs that encounter endplate fractures may need treatment like other fracture sites. An endplate fracture could be treated with initial unloading followed by gradual reloading of the disc to give the endplate enough healing time to withstand minimal compression forces (Adams et al., 2010).

\section{Conclusion}

When examining damage to disc tissues using experimental models, high torsion loads, multiplanar movements, and cyclical movement in the sagittal plane with axial compression were shown to subject the annulus and endplate of an intervertebral disc to injury. Pressure placed on annular fibers from within or through axial compression cause fissures often beginning mid-annulus and initially progress inwardly, then posterior-laterally. Sudden, high-compression axial loads made discs susceptible to herniations and endplate fractures.

To improve the effectiveness and expedite return to activity for athletes with disc injuries, initially restrict movement and temporarily prohibit axial compression if endplate fractures are suspected. Specific, nonaggressive movements for disc dehydration, fibroblast proliferation, postural awareness, and scarring prevention between annular layers can be administered early. Sub-acutely strategies to facilitate the diffusion of nutrients through the endplate into the nucleus could 
be warranted in a graded fashion of intensity. Lumbar segmental stability may contribute to protection via shear force restraint and encouraging rotational mobility from the thoracic region and internal rotation of the hips with athletic movements.

A direct link between external forces created by athletic movements and precise injury to the disc using human specimens would give a more accurate indication of extent and types of injury. More outcome data on effectiveness of treatment strategies specific to the unique tissues representing intervertebral discs and facilitation of nutrition with human disc is needed.

\section{References}

Adams, M. A. \& Dolan, P. (2016). Lumbar intervertebral disk injury, herniation and degeneration. In: Pinheiro-Franco JL, ed. Advanced concepts in lumbar degenerative disk disease. Springer-Verlag Berlin Heidelburg, 23-39.

Adams, M. A. \& Hutton, W. C. (1983). The effect of posture on fluid content of lumbar intervertebral discs. Spine, 8, 665-671.

Adams, M. A., Dolan, P. \& Hutton, W. C. (1987). Diurnal variations in the stresses on the lumbar spine. Spine, 12, 130-137.

Adams, M. A., Stefanakis, M. \& Dolan, P. (2010). Healing of a painful intervertebral disc should not be confused with reversing disc degeneration: implications for physical therapists for discogenic back pain. Clinical biomechanics, 25, 961-971.

Ahlgren, B. D., Lui, W., Herkowitz, H. N., Panjabi, M. M. \& Guiboux, J. P. (2000). Effect of annular repair on the healing strength of the intervertebral disc: a sheep model. Spine, 25, 2165-2170.

Ahlgren, B. D., Lui, W., Herkowitz, H. N., Panjabi, M. M. \& Guiboux, J. P. (2000). Effect of anular repair on the healing strength of the intervertebral disc: a sheep model. Spine, 25, 2165-2170.

Beattie, P. F., Donley, J. W., Arnot, C. F. \& Miller, R. (2009). The change in the diffusion of water in normal and degenerative lumbar intervertebral discs following joint mobilization compared to prone lying. The Journal of orthopaedic and sports physical therapy, 39, 4-11.

Beeckmans, N., Vermeersch, A., Lysens, R., Wambeke, P. V., Goossens, N., Thys, T., Brumagne, S. \& Janssens, L. (2016). The presence of respiratory disrorders in individuals with low back pain: a systematic review. Manual therapy, 26, 77-86.

Berger-Roscher, N., Casaroli, G., Rasche, V., Villa, T., Galbusera, F. \& Wilke, H. J. (2017). Influence of complex loading conditions on intervertebral disc failure. Spine, 424, E78-E85.

Bogduk, N., Tynan, W. \& Wilson, A.S. (1981). The nerve supply to the human lumbar intervertebral discs. Journal of anatomy, 132, 39-56.

Brisby, H., Wei, A. Q., Molloy, T., Chung, S. A., Murrell, G. A. \& Diwan, A. D. (2010). The effect of running exercise on intervertebral disc extracellular matrix production in a rat model. Spine, 35, 1429-1436.

Callaghan, J. P. \& McGill, S. M. (2001). Intervertebral disc herniation: studies on a porcine model exposed to highly repetitive flexion/extension motion with compressive force. Clinical biomechanics, 16, 28-37.

Curry, W. H., Pintar, F. A., Doan, M. B., Nguyen, H. S., Eckardt, G., Baisden, J. L., Maiman, D. J., Paskoff, G. R., Shender, B. S. \& Stemper, B. D. (2016). Lumbar spine endplate fractures: biomechanical evaluation and clinical considerations through experimental induction of injury. Journal of orthopaedic research, 34, 1084-1091.

Dines, J. S., Bedi, A., Williams, P. N., Dodson, C. C., Ellenbecker, T. S., Altchek, D. W., Windler, G., \& Dines, D. M. (2015). Tennis injuries: epidemiology, pathophysiology, and treatment. The Journal of the American Academy of Orthopaedic Surgeons, 20, 181-189.

Dowdell, J., Mikhail, C., Robinson, J. \& Allen, A. (2018). Anatomy of the pediatric spine and spine injuries in young athletes. Annals of Joint, 3, 1-10. 
Ehrlich, G. E. (2003). Low back pain. Bull World Health Organ, 81, 671-676.

Fardon, D. F., Williams, A. L., Dohring, E. J., Murtagh, F. R., Rothman, S. L. G. \& Sze, G. K. (2014). Lumbar disc nomenclature: version 2.0 recommendations of the combined task forces of the north American spine society, the American society of spine radiology and the American society of neuroradiology. The spine journal, 14, 2525-2545.

Fleisig, G. S., Hsu, W. K., Fortenbaugh, D., Cordover, A., \& Press, J. M. (2013). Trunk axial rotation in baseball pitching and batting. Sports Biomech, 12, 324-333

Fu, M. C., Ellenbecker, T. S., Renstrom, P. A., Windler, G. S., \& Dines, D. M. (2018). Epidemiology of injuries in tennis players. Current reviews in musculoskeletal medicine, 11, 1-5.

Gatt, C. J., Hosea, T. M., Palumbo, R. C., \& Zawadsky, J. P. (1997). Impact loading of the lumbar spine during football blocking. The American journal of sports medicine, 25, 317-321.

Gray, B. L., Buchowski, J. M., Bumpass, D. B., Lehman, R. A., Mall, N. A., \& Matava, M. J. (2013). Disc herniations in the national football league. Spine, 38, 1934-1938.

Hangai, M., Kaneoka, K., Hinotsu, S., Shimizu, K., Okubo, Y., Miyakawa, S., Mukai, N., Sakane, M., \& Ochiai, N. (2009). Lumbar intervertebral disk degeneration in athletes. The American journal of sports medicine, 37, 149-155.

Hsu, W. K., McCarthy, K. J., Savage, J. W., Roberts, D. W., Roc, G. C., Micev, A. J., Terry, M. A., Gryzlo, S. M. \& Schafer, M. F. (2011). The professional athlete spine initiative: outcomes after lumbar disc herniation in 342 elite professional athletes. The spine journal, 11, 180-186.

Huang, C. Y. \& Gu, W. Y. (2008). Effects of mechanical compression on metabolism and distribution of oxygen and lactate in intervertebral disc. Journal of biomechanics, 41, 1184-1196.
Hughes, S. P. F., Freemont, A. J., Hukins, D. W. L., McGregor, A. H. \& Roberts, S. (2012). The pathogenesis of degeneration of the intervertebral disc and emerging therapies in the management of back pain. The Journal of bone and joint surgery, 94, 1298-1304.

Iwamoto, J., Sato, Y., Takeda, T. \& Matsumoto, H. (2010). The return to sports activity after conservative or surgical treatment in athletes with lumbar disc herniation. American journal of physical medicine \& rehabilitation, 89, 1030-1035.

Kingsley, M. I., D’Silva, L. A., Jennings, C., Humphries, B., Dalbo, V. J. \& Scanlan, A. T. (2012). Moderate-intensity running causes intervertebral disc compression in young adults. Medicine and science in sports and exercise, 44, 2199-2204.

Krismer, M., Haid, C. \& Rabl, W. (1996). The contribution of annulus fibers to torque resistance. Spine, 21, 2551-2557.

Kulling, F. A., Florianz, H., Reepschlager, B., Gasser, J., Jost, B., \& Lajtai, G. (2014). High prevalence of disc degeneration and spondylolysis in the lumbar spine of professional beach volleyball players. Orthopaedic journal of sports medicine, 2, 1-6.

Latham, J. M., Pearcy, M. J., Costi, J. J., Moore, R., Fraser, R. D. \& Vernon-Roberts, B. (1994). Mechanical consequences of annular tears and subsequent intervertebral disc degeneration. Clinical biomechanics, 9, 211-219.

Lee, C. Y., Liu, X., Smith, C. L., Zhang, X., Hsu, H. C., Wang, D. Y. \& Luo, Z. P. (2004). The combined regulation of estrogen and cyclic tension on fibroblast biosynthesis derived from anterior crutiate ligament. Matrix biology, 23, 323-329.

Lindsay, D. M., \& Vandervoort, A. A. (2014). Golf-related low back pain: a review of causative factors and prevention strategies. Asian journal of sports medicine, 5, 1-8.

Lurie, J. D., Tosteson, T. D., Tosteson, A. N. A., Zhao, W., Morgan, T. S., Abdu, W. A., Herkowitz, H. \& Weinstein, J. N. (2014). Surgical versus 
nonoperative treatment for lumbar disc herniation: eight-year results for the spine patient outcomes research trial (SPORT). Spine, 39, 3-16.

Malko, J. A., Hutton, W. C. \& Fajman, W. A. (2002). An in vivo MRI study of the changes in volume (and fluid content) of the lumbar intervertebral disc after overnight bed rest and during an 8-hour walking protocol. Clinical spine surgery, 15, 157-163.

Mundt, D. J., Kelsey, J. L., \& Golden, A. L. (1993). An epidemiological study of sports and weight lifting as possible risk factors for herniated lumbar and cervical discs. The American journal of sports medicine, 21, 854-860.

Noguchi, M., Gooyers, C. E., Karakolis, T., Noguchi, K. \& Callaghan, J. P. (2016). Is intervertebral disc pressure linked to herniation?: An in-vitro study using porcine model. Journal of biomechanics, $\mathbf{4 9}$, 1824-1830.

Rajasekaran, S., Bajaj, N., Tubaki, V., Kanna, R. \& Shetty, A. P. (2013). ISSLS prize winner: the anatomy of failure in lumbar disc herniation: an in vivo, multimodel, prospective study of 181 subjects. Spine, 38, 1491-1500.

Schmidt, H., Kettler, A., Heuer, F., Simon, U., Claes, L. \& Wilke, H. J. (2007). Intradiscal pressure, shear strain, and fiber strain in the intervertebral disc under combined loading. Spine, 32, 748-755.

Shan, Z., Wade, K. R., Schollum, M. L., Robertson, P. A., Thambyah, A. \& Broom, N. D. (2017). A more realistic disc herniation model incorporating compression, flexion and facet-constrained shear: a mechanical and microstructural analysis. Part II: high rate or 'surprise' loading. European spine journal, 26, 2629-2641.

Tampier, C., Drake, J. D., Callaghan, J. P. \& McGill, S. M. (2007). Progressive disc herniation: an investigation of the mechanism using radiologic, histochemical, and microscopic, dissection techniques on a porcine model. Spine, 32, 2869-2874.

Trompeter, K., Fett, D. \& Platen, P. (2017). Prevalence of back pain in sports: a systematic review of the literature. Sports medicine, 47, 1183-1207.

van Heeswijk, V. M., Thambyah, A., Robertson, P. A., \& Broom, N. D. (2017). Posterolateral disc prolapse in flexion initiated by lateral inner annular failure: an investigation of the herniation pathway. Spine, 42, 1604-1613.

Veres, S. P., Robertson, P. A. \& Broom, N. D. (2008). ISSLS prize winner: microstructure and mechanical disruption of the lumbar disc annulus: part II: how the annulus fails under hydrostatic pressure. Spine, 33, 2711-2720.

Veres, S. P., Robertson, P. A. \& Broom, N. D. (2009). The morphology of acute disc herniation: a clinically relevant model defining the role of flexion. Spine, 34, 2288-2296.

Veres, S. P., Robertson, P. A. \& Broom, N. D. (2010a). ISSLS prize winner: how loading rate influences disc failure mechanics: a microstructural assessment of internal disruption. Spine, 35, 1897-1908.

Veres, S. P., Robertson, P. A., Broom, N. D. (2010b). The influence of torsion on disc herniation when combined with flexion. European spine journal, 19, 1468-1478.

Videman, T., Gibbons, L. E., Kaprio, J. \& Battie, M. C. (2010). Challenging the cumulative injury model: positive effects of greater body mass on disc degeneration. The spine journal, 10, 26-31

Wade, K. R., Robertson, P. A., Thambyah, A. \& Broom, N. D. (2014). How healthy discs herniate: a biomechanical and microstructural study investigating the combined effects of compression rate and flexion. Spine, 39, 1018-1028.

Wade, K. R., Robertson, P. A., Thambyah, A. \& Broom, N. D. (2015). 'Surprise' loading in flexion increases the risk of disc herniation due to annulus-endplate junction failure: a mechanical an microstructural investigation. Spine, 40, 891-901.

Wade, K. R., Schollum, M. L., Robertson, P. A., Thambyah, A. \& Broom, N. D. (2017). A more realistic disc herniation model incorporating 
compression, flexion and facet-constrained shear: a mechanical and microstructural analysis. Part I: low rate loading. European spine journal, 26, 2616-2628.

Wang, H., Cheng, J., Xiao, H., Li, C. \& Zhou, Y. (2013). Adolescent lumbar disc herniation: experience from a large minimally invasive treatment centre for lumbar degenerative disease in Chongping. China. Clinical neurology and neurosurgery, 115, 1415-1419.

Wang, Y., Videman, T. \& Battie, M. C. (2012). ISSLS prize winner: lumbar vertebral endplate lesions: associations with disc degeneration and back pain history. Spine, 37, 1490-1496. 